\title{
The research status and prospects of the econopower car
}

\author{
Luo Jin, Ma Qihua, Luo Yiping
}

Faculty of Automotive Engineering, Shanghai University of Engineering and Science, Shanghai, China

\section{Email address:}

84276661@qq.com (Luo Jin), mqh0386@163.com (Ma Qihua), lyp777@ sina.com (Luo Yiping)

\section{To cite this article:}

Luo Jin, Ma Qihua, Luo Yiping. The Research Status and Prospects of the Econopower Car. International Journal of Energy and Power Engineering. Vol. 4, No. 1, 2015, pp. 11-14. doi: 10.11648/j.ijepe.20150401.12

\begin{abstract}
Since 2007, Honda China Eco Mileage Challenge was held for 7 years, so far, nearly hundred teams participated in the competition. Although the national ranking of our school's fuel econopower cars is ideal, but the competition results between our school and high level universities is remote. For raising the grade of the econopower car, this paper analyzed the articles about econopower car that published in recent years, discusses the main research direction of econopower car and main achievements, analyse it from the main research direction and research method, offer the technology of econopower car at current in domestic, summarizes the research trend of econopower car, make guidance for the future design of econopower car, also.
\end{abstract}

Keywords: Econopower Car, Optimization, Summarization

\section{Introduction}

Throughout the domestic study of econopower cars, mainly in the reform of the engine and the optimization design of frame, body, and each system. The engine is the soul of the econopower car, engine optimization results directly determines the final score of the competition. But only good engine is not enough, the simulation analysis in theory and actual combat prove, lightweight and streamline of car body frame is the most important aspects of econopower car. At the same time, the optimization design of driving, steering and braking system of econopower car is indispensable, also.

\section{The Optimal Design of the Frame}

The optimization design of the frame is such an eternal theme of econopower cars, mainly on lightweight of the frame, mainly divided into structural lightweight and materials lightweight, the main method is using finite element analyse the characteristics, such as frame stiffness, strength and modal, which to provide theoretical basis for improving design of frame structure.

\subsection{The Optimal Design of Frame Structure}

Shanghai university of engineering science ${ }^{[1]} \mathrm{Wu}$ Hao is proposed from the mechanics, with the beam frame (as shown in figure 1) as a starting point, design a new frame structure, decide made the frame structure to the space structure ,finally (as shown in figure 2). Beijing Vocational College of
Electronic Science ${ }^{[2]} \mathrm{Li}$ Xiao et al. proposed that adopts industrial aluminum alloy cold drawn pipe welding in frame deformation of bearing part. Nanjing Agricultural University ${ }^{[3]}$ Wu Luning proposed add 2 oblique steel pipe in the middle part of the frame, finally get the maximum deformation of frame is $0.33 \mathrm{~mm}$, the maximum stress is $23 \mathrm{Mpa}$, far less than the material yield strength. Taiyuan University of Science and Technology $y^{[4]} \quad$ Yang Chong put forward adopts the edge beam type parallel trapezoidal structure in the frame, and optimize the frame of section size ,made the local deformation of the frame and the stress concentration improved obviously, the frame portion weight loss $16.96 \%$.

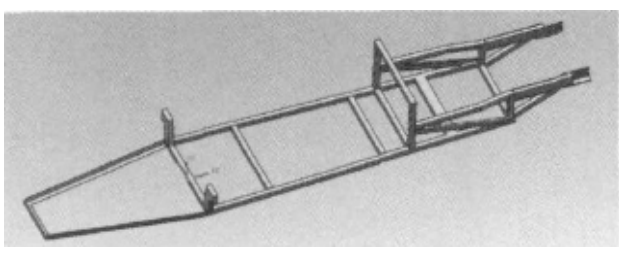

Fig. 1. The fringe beam frame.

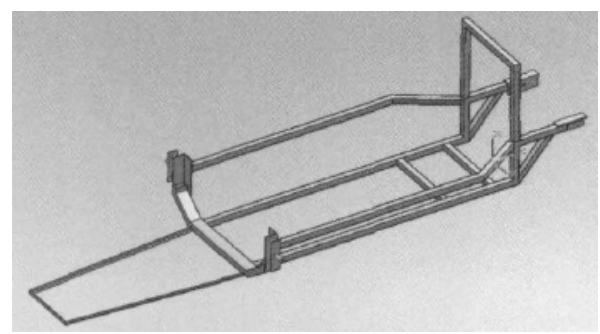

Fig. 2. The spatial structure of the frame. 


\subsection{The Optimal Design of Frame Material}

For the aspect of material optimization, the research of current domestic in material for the frame focused on metal materials, the main method is to use the finer rod instead of the before rod or using smaller density metal materials to replace the original materials. Such as the reference [1] from the strength, stiffness, quality and efficiency of the frame to selected thin-walled square steel of $1 \mathrm{~mm}$ wall thickness Q235-A as the frame material. Reference [2] consider many factors finally selected hard alloy for frame materials. From reference [3] after taken into consideration and selected $25 *$ $38 \mathrm{~mm}$ stainless steel square tube as the frame material. Reference [4] frame materials selected of AL6061 aluminum alloy pipe.

\subsection{Summary}

The optimal design of the frame focuses on the optimization of the structure and to select the smaller density metal material as the material of frame, rarely involves non-metallic materials, namely using non-metallic materials on the frame. According to results of the teams for the frame optimization show that structural optimization has been basically meet the requirements, and non-metallic materials such as carbon fiber have the advantages that not only light ,but also can absorb bump energy. So the frame of the research trend in the future tend to under the circumstances that guarantee the frame of various aspects performance all meet the requirements, applying non-metal materials to the econopower car frame to achieve the goal of lightweight.

\section{Optimal Design of Car Body}

The optimal design of econopower car body is another important task, including the optimization of outer body and internal body, mainly streamlined the outer body in domestic and ignored the optimization design of internal car body.

\subsection{The Optimal Design of the Outer Car Body}

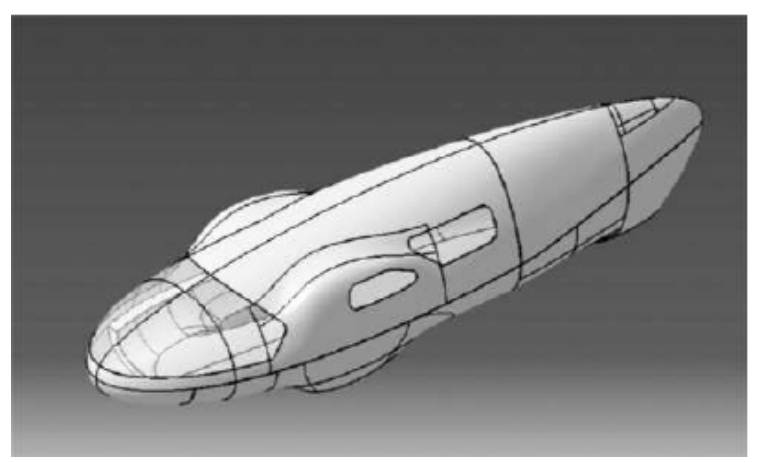

Fig. 3. The body model of airfoil curve.

The current trend of car body research focused on the optimization design of outer car body in domestic, Taiyuan university of science and technology ${ }^{[4]}$ Yang Chong put forward on the basis of pro/e software, using the ISDX module to reverse development of econopower car body, complete a body design with the goal of minimizing air resistance streamlined. Tongji university ${ }^{[5]} \mathrm{Li}$ Bowen put forward the airfoil or truncate the airfoil in status type surface, in the body, the shell ridge line to optimize the vehicle's aerodynamic performance (as shown in figure 3). Beijing forestry university ${ }^{[6]}$ Lan Baozhen developed a follow-up tail system, and through a closed loop control system to adjust the wing angle and tail gap to meet the performance requirements.

\subsection{The Optimal Design of Internal Car Body}

At present, the researchers ignored the fact that the structure of the cockpit is also decided the body shape, if outer car body were optimized design, and neglect the arrangement of the internal components, made the outer car body presents a streamlined is important, but its internal structure can also affect the driver's operation space and driving comfort which affects the results of the competition. Hubei Industry University ${ }^{[7]}$ ChuYiYi proposed to carry out ergonomics optimization design in the working area of the econopower car, but the reference is just put forward suggestion, did not have the actual work.

\subsection{Summary}

The current research on econopower car body focus on the outer body in domestic, ignored the design of the internal car body, as is known to all, if the arrangement of the cockpit components is not reasonable, it can make the driver feel inconvenience or lack of comfort, ultimately influence the challenge performance. To be let the assignments section of the econopower car for the ergonomics optimization, make the internal structure is compact but drivers have enough space to operate, at the same time, to aerodynamic optimize the outer body of the econopower car.

\section{The Improvement of Engine}

From the results of the performance of fuel group econopower car, improve the engine is one of the main measures to improve cars' performance. In recent years, the literatures about improve engine tend to optimization of engine inlet and exhaust system, optimization of ignition timing and combustion space, engine lightweight.

\subsection{Optimization of Inlet and Exhaust System}

For engine, the optimization of inlet and exhaust system is one of the main work, make more fresh air into and make more exhaust gas out can make the combustion more sufficient and releases more energy. Wuhan technology university ${ }^{[8]} \mathrm{Xia}$ Xiaokun put forward the idea that changed the main hole of the carburetor, reducing fuel and air mixing ratio to make the fuel burn adequately, at the same time, cover insulation material on the intake pipe and cylinder, so that even a relatively thin mixture, fuel can fully burning. Shanghai university of engineering science ${ }^{[9]} \mathrm{Li}$ Haifeng et al. put forward thickening intake pipe to remove the pressure from the crankcase and reduce the motion resistance of the crankshaft. 


\subsection{Optimization of Ignition Timing and combustion Space}

Beijing technology university ${ }^{[10]} \mathrm{Hu}$ Meiqi adopt the method of laser welding weld a piece of aluminum piston with the original piston, to improve the fuel economy of the engine (as shown in figure 4). Beijing technology university ${ }^{[11]}$ Liu Jun using particle swarm optimization, from the theory of optimum to design optimal number of ignition and optimal ignition position during the whole process, so as to enable the econopower cars can not only complete total distance, but also use the theory minimum of oil within the prescribed period of time.

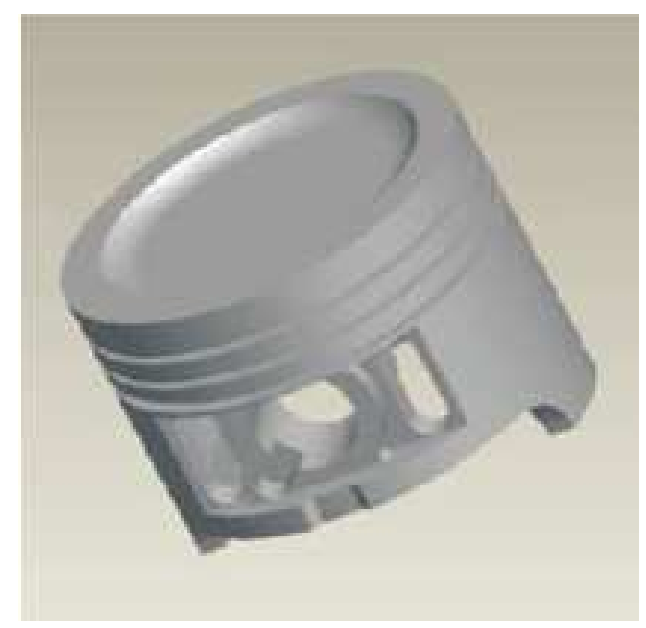

(a)The original piston

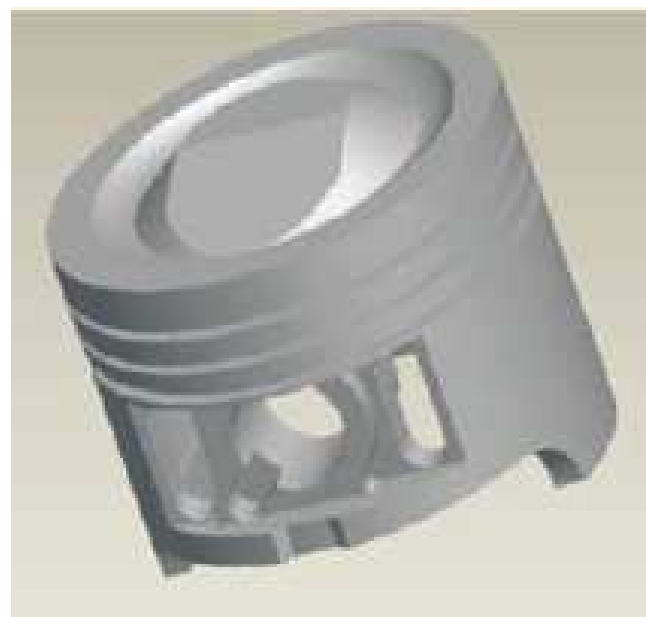

(b)The piston welding a block

Fig. 4. The optimization of the piston.

\subsection{Lightweight Engine}

Lightweight and conducting technical reforming on the engine, reducing engine friction resistance. Wuhan technology university $^{[8]}$ Xia Xiaokun puts forward to remove magneto coil and other spare parts to lightweight the engine. Hubei University of Automotive Technology ${ }^{[12]}$ Wang Xin proposed to reduce the weight of the engine by remove the start machine parts of the engine and the scales distribution around the cylinder.

\subsection{Summary}

The current research on improvement of econopower car engine in domestic, most only based on a certain parts or aspects and still stay in the experience, did not consider the multiple parts or aspects influence on engine performance, also lack of guidance of software. The future research trend of the improvement of engine will focus on multiple parts or aspects through software simulation, obtained energy saving effects of the revamp, confirmed its energy saving effect is better than the before engine, it will be executed.

\section{Other System Optimal Design of Econopower Car}

In addition to the above three main approaches to improve the performance of econopower car, the econopower team improve the car mainly through optimal design of the drive, steering and braking system to improve the dynamic performance and economic performance of econopower cars.

\subsection{Optimal Design of Steering and Braking System}

Chang an university Zhao Yan ${ }^{[13]}$, Lei Lei ${ }^{[14]}$ make use of ADAMS software to entity modeling and correction of movement about the steering system to verified the rationality of the steering mechanism.

The brake system of econopower car is commonly using bicycle manually brake system, in the case of meet the braking performance using light quality brake system, so as to achieve the goal of saving fuel.

\subsection{Optimal Design of Driving System}

Traditional forms of automobile transmission are shaft, chain and belt transmission. In order to reduce vehicle weight, use chain or belt with light materials to transmit engine power [13] [14].

There are two kinds of typically wheel configuration: front two wheels, rear one wheel ${ }^{[2][13][14]}$ and front one wheel, rear two wheels. The former has the advantage of simple structure, easily to manufacture, small resistance, good stability and without differential part, etc. The latter has the advantage that can use bicycle tire, reducing wheel weight, reduce the friction resistance of racing cars.

\subsection{Summary}

Though this part optimization of econopower car in domestic is less, whether the system of steering and braking is flexible, whether the selection of tire and ratio of the transmission is reasonable, whether the assembly location of engine and install posture is proper are will affect the performance of econopower car. Therefore, it is to be further optimization of these systems in the follow-up work.

\section{Summary}

So far, the optimal design of econopower car is priority to 
frame, engine, car body, complementary to steering, transmission system, making them cooperate with each other, to make the econopower car has a better economic performance and dynamic performance,

From the references of recent years, it seems, the optimization of econopower car also exist a few defects: the optimization of the frame has focused on the structure light and ignore the material light, the optimization of car body has focused on the outer body and neglected the inner body, influence on engine performance are various, most current references is only based on one aspect or a part of engine, and the optimization of the engine is based on experience, lack of theoretical guidance.

So, the research trend in the future will focus on the above defects to further optimize, namely: apply nonmetallic materials on econopower car frame to achieve lightweight, streamline the outer car body, at the same time to ergonomics optimal design of econopower car assignments section, on the basis of the simulation software rather than experience to modify the engine. If can achieve breakthrough in these aspects, our school will be able to achieve better results in Honda China Eco Mileage Challenge.

\section{References}

[1] Wu Hao. Frame design and finite element analysis of electric econopower car $[\mathrm{J}]$. Technology of vehicle and power, 2013, (2): $28-30$.

[2] Li Xiao, Zhu Xun, Guan Xiaoqing. Econopower car frame structure optimization design based on finite element analysis [J]. Journal of computer applications and software, 2013, (10) : 99-101.

[3] Wu Luning, Lu Zhixiong, Yang Huiliang, Xia Yunhao. Honda econopower car frame optimal design based on ANSYS Workbench [J]. Journal of agricultural equipment and vehicle engineering, 2014, 52 (2) : 14-17.
[4] Yang Chong. Frame lightweight and body dynamics research of econopower car [D]. Taiyuan, Taiyuan university of science and technology, 2014.

[5] Li Bowen. body development of econopower car based on CFD [J]. Journal of automobile engineering, 2013, (4): 37-41.

[6] Lan Baozhen, Chen Shao, Sun Xiaotian. Servo tail oil-saving technical analysis of Econopower cars based on CFD [J]. Journal of automobile engineering, 2014, (4): 17-22.

[7] ChuYiyi,Tang Dehong. The research of assignments section of econopower car based on ergonomics [J]. Journal of design, 2014, (3) : 190-191.

[8] Xia Xiaokun, Yan Fuwu, Du Changqing. Research of reduce fuel consumption rate of econopower car [J]. Journal of motorcycle technology, 2008, (10): 70-72.

[9] Li Haifeng, Zhu Tianxiang, Li Wei. The optimization of Econopower car air intake system [J]. Journal of engineering and technology, 2013, (11): 25-26.

[10] Hu Meiqi, Duan Yan, Chen Yinan, Zhang Youtong. Improvement design of econopower car engine piston [J]. Journal of automotive engineering, 2011, (10) : 35 to 37

[11] Liujun, Ren Xuemei, Ma Hongbin. The optimal design of high efficiency and econopower car ignition time [J]. Journal of Beijing technology university, 2013, (6): 586-589.

[12] Wang Xin, Gou Zhibin, Zheng Sizhe, Yan Shiming. Improvement of econopower car engine performance $[\mathrm{J}]$. Journal of Hubei University of Automotive Technology, 2011, 25 (2): 75-80.

[13] Lei Lei. Research of econopower car research technology [D]. Xi 'an: Chang 'an university, 2008.

[14] Zhao yan. Research and development of econopower car and research of technology [D]. Xi 'an: Chang 'an university, 2009. 